\title{
Effect of Internal Direct and Inverted Alu Repeat Sequences on PCR
}

\author{
Weizhen Ji, Xian-Yang Zhang, G. Sakuntala Warshamana, Guang-zhi Alan Qu, and \\ Melanie Ehrlich
}

Department of Biochemistry, Tulane Medical School, New Orleans, Louisiana 70112

\begin{abstract}
We have studied the effect of repeated DNA sequences, especially Alu repeats, on PCR. Alu repeats are sequences that are $\sim 300$ bp long and interspersed at a very high copy number throughout the human genome. We amplified part of the human lowdensity lipoprotein receptor gene containing two Alu repeat sequences in the same orientation, $\sim 7.8 \mathrm{~kb}$ apart, with unique sequence primers outside these repeats. The major PCR product was a DNA fragment with an in vitro deletion between the Alu repeats. The formation of this product depended on the template concentration and the type of polymerase used. Such a product arose apparently as a result of a "jumping reaction" involving a primer whose extension was terminated prematurely within one Alu repeat followed by annealing of such an incompletely extended primer to the other, distant Alu repeat. No such jumping products were seen when a $0.8-k b$ region containing two nearby inverted Alu repeats within the human $\alpha$-galactosidase A gene was subject to PCR with unique sequence primers annealing just outside these repeats.
\end{abstract}

uring PCR, unexpected products can arise because of incomplete extension of one or both unique-sequence primers on templates containing allelic sequences. ${ }^{(1)}$ Thus, two alleles on different DNA molecules can become joined to give a hybrid gene, ${ }^{(1,2)}$ especially when short primer elongation times are employed. ${ }^{(3)}$ Upon PCR, the direct repeats in a human minisatellite DNA template can lead to a heterodisperse smear in the late cycles, which could be attributable to out-of-register annealing of the singlestranded repeated sequences. ${ }^{(4)}$ In the case of inverted repeats amplified by PCR, evidence for another artifact has been presented, namely the formation of fold-back structures read through by the DNA polymerase. ${ }^{(5)}$

We were interested in developing PCR assays for detecting very low numbers of chromosomes containing rearrangements at human Alu repeats, a family of interspersed repeated sequences of $\sim 300$ bp present in $\sim 500,000$ copies in the human genome. ${ }^{(6,7)}$ Alu repeats generally display $\sim 70-90 \%$ homology to one another. Some appear to predispose DNA to illegitimate recombination events, such as deletions and inversions. ${ }^{(8-12)}$ In this report we describe a PCR artifact giving rise to a deletion in vitro from Alu repeats present in the same orientation $\sim 7.8 \mathrm{~kb}$ apart within the human low-density lipoprotein receptor (LDLR) gene ${ }^{(12)}$ and have examined various conditions to try to minimize this artifact. The deletion product obtained in vitro is the same as a naturally occurring in vivo deletion. ${ }^{(12)}$ In contrast, no analogous PCR artifact was seen with inverted Alu repeats present 145 bp apart in the human $\alpha$-galactosidase A $(\alpha$-gal $) .{ }^{(13)}$ gene upon amplification for 46 cycles.

\section{MATERIALS AND METHODS}

Template DNA

Genomic DNA was isolated by standard methods $^{(14)}$ from human brain or intestine, HeLa cells, human cell lines (GM04312A, GMO80505, and GM00637F; Coriell Institute), or rat spleen. The source of the LDLR gene deletion of $\sim 7.8$ $\mathrm{kb}$ was plasmid pLDLR-FH781 (Fig. $1),{ }^{(12)}$ containing a $10-\mathrm{kb} \mathrm{XbaI}$ fragment from intron 12 to the end of exon 18. For analysis of PCR through a region with nearby 30-bp inverted repeats, the plasmid pMKIVdel was constructed. This 30bp sequence, from position +93 to +122 of the herpes simplex virus thymidine kinase (HSV TK) gene, was inserted via BstEII and KpnI overhangs in inverse orientation into a 5.5-kb KpnI-BstEII partial digestion fragment from $\mathrm{pMK}^{\prime}$, an HSV TK expression plasmid. ${ }^{(15)}$ The pMKIVdel DNA also contained a 411-bp deletion from the BglII site to the BstEII site between the copies of the 30-bp inverted repeat to leave a 28 -bp gap between these repeats.

\section{Primers for PCR}

Primers for the antisense strand of exon 18 of the human LDLR gene were as follows: ex18a, 5'-AAACGATCCAGACTGGAGG-3' (32 nucleotides downstream of Alu repeat 3 in exon 18; Fig. 1) and ex18b, 5'-TCAGAAAGAACCTGAAGTCCCGTC-3' (beginning 1 nucleotide downstream of the end of ex18a). The sense strand primers from intron 15 were as follows: in 15c, 5'-GGATTCCTGGGCAGGGCACA-3' (including 8 nucleotides of the $5^{\prime}$ end of an Alu repeat; Fig. 1); ${ }^{(12)}$ in $15 b, 5$ '-CTGGGCAACATAGCGAGAC-3' ( 60 nucleotides upstream of in $15 \mathrm{c}$ ); and in $15 \mathrm{a}$, 
A

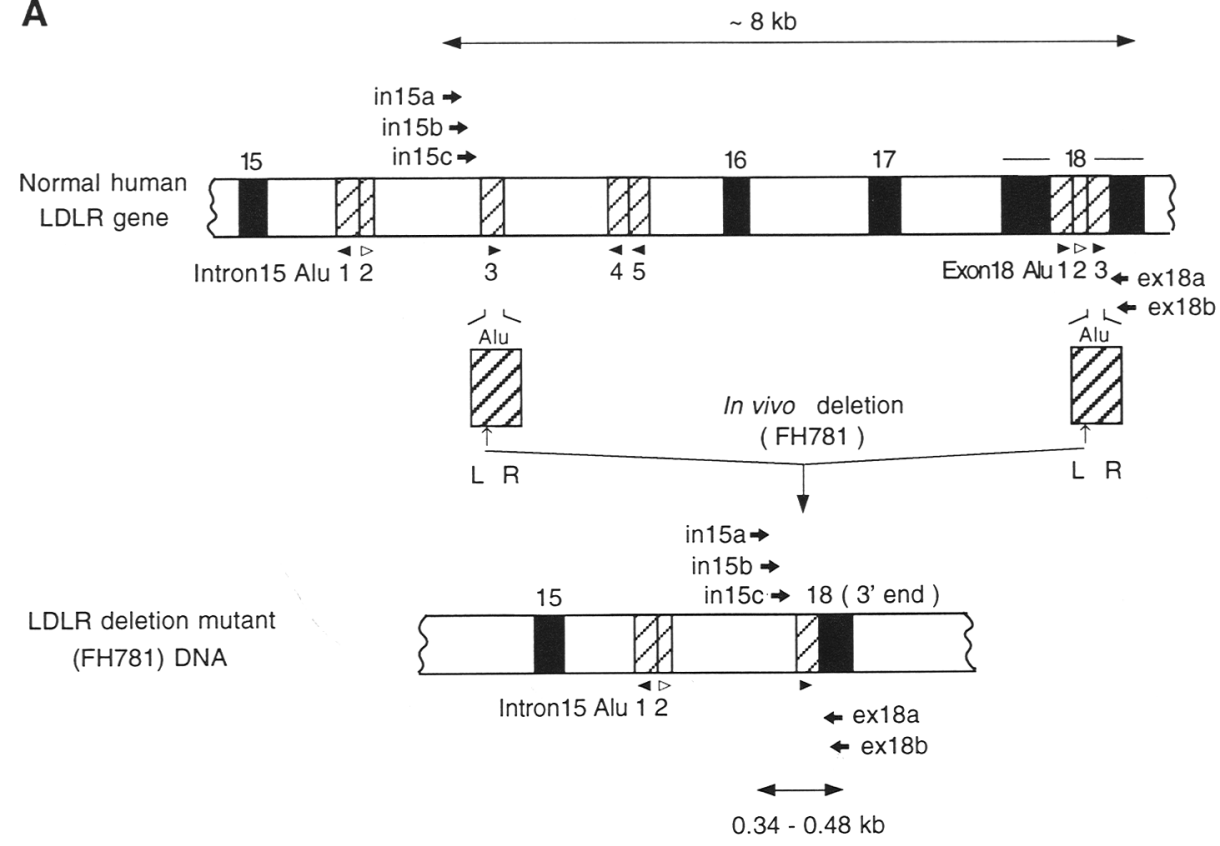

B

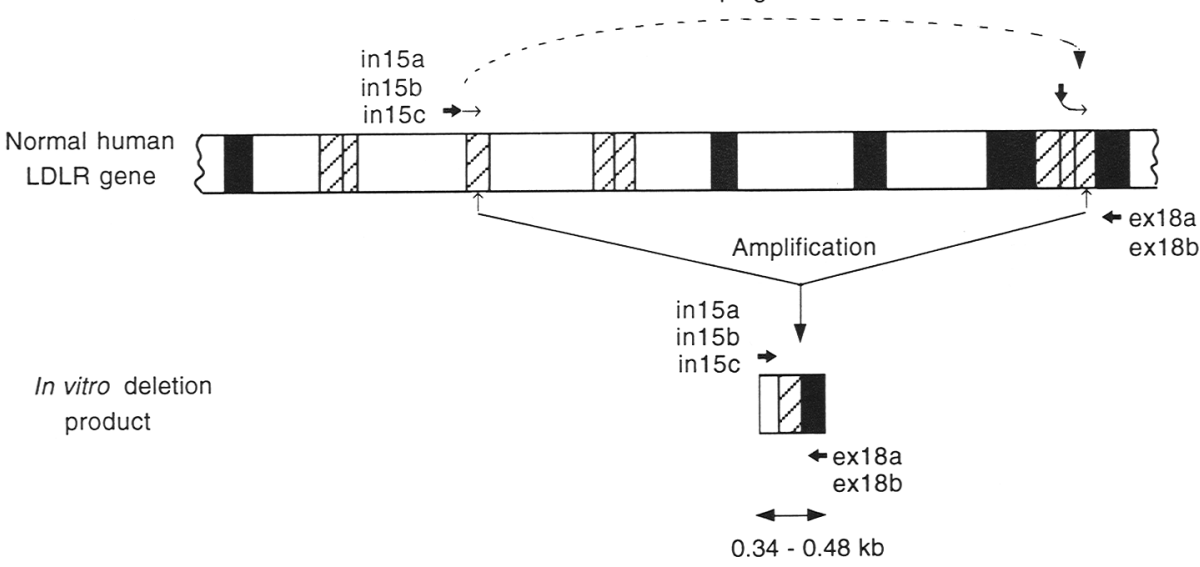

FIGURE 1 Scheme for PCR detection of deletion mutations in the LDLR gene and for the generation of an in vitro deletion product by a jumping reaction. $(A)$ The human LDLR gene from the end of intron 14 and to the beginning of intron 18 is shown (not to scale) for both the normal and FH781 deletion mutant allele ${ }^{(12)}$ as well as an enlarged version of the two Alu repeats involved in the deletion and the position of the deletion junction. (Solid bars) exons; (open segments) introns; (short arrows) nonoverlapping primers; (hatched bars) Alu repeats; (directionality: solid arrowheads for complete repeat sequences; open arrowheads for partial repeats). ( $L$ and $R$ ) Left and right monomers, respectively, in Alu repeats, which typically are head-to-tail dimers. The expected range of sizes of PCR products that should be obtained with different combinations of primers is indicated by double-headed arrows. $(B)$ One likely pathway for generation of the in vitro PCR deletion product. The incompletely extended PCR primers are indicated by contiguous thick and thin arrows. The distances between sense strand and antisense strand primers are shown by a double-headed arrow. It is equally likely that the jumping reaction could occur with incompletely extended exon 18 primers rather than intron 15 primers as shown.

5'-CCTAGCTACTCCCAGAGGCTGAG-3' $(\sim 120$ nucleotides upstream of in $15 \mathrm{c})$. To design primers in $15 a$ and in $15 b$, first we had to sequence part of intron $15 \mathrm{im}$ mediately upstream of its Alu repeat 3
(Fig. 1) using PLDLR-FH781 as the template. The deduced sequence $\sim 20$ bp from the sequencing primer (5'-GCCCTGCCCAGGAAT- $3^{\prime}$ ) is as follows: $5^{\prime}$-TTTTTATTTTTAGAGATGGGGTCTCGCTATG-
TTGCCCAGGCTGGACTTCAACTCTTGGGCTCAAGCAAGTCTCCCGCCTCAGCCTCTGGAGTAGCTAGGACTGCAGGCGTGCACCTCCACACCAG-3'.

For amplifying the human $\alpha$-gal gene, we used the following sense-strand primers with the position numbers in the gene $^{(16)}$ indicated: a, $5^{\prime}$-GAGTACCTCTGCATTGATGACTGTTG-3', , (5158-5183); b, 5'-CAGAGGACTGTAAGCACTTCTGTA-3' (5299-5322). The antisense strand primers were as follows: c, 5'-CAAAAGGAGAACTCCAAGATGG-3' (6111-6090) and d, 5'-CATGCACACATATGTCAAGAACCA-3' (6172-6149). For PCR on the inverted repeat region in pMKIVdel, we used primers 5'-GGCATAATAGACATATTGCTGGTGGTGG-3' (from the sense strand, 255 nucleotides upstream of the first 30-bp repeat) and 5'-AACGCAGACGCGTGTTGATGGCA-3' (43 nucleotides downstream of the second 30-bp repeat), which should give a 386-bp product.

\section{PCR Amplification Conditions}

PCR was carried out in a thermal cycler (Ericomp) using either Taq polymerase (Promega), AmpliTaq Stoffel fragment (Perkin-Elmer), Vent polymerase, Vent exo $^{-}$(New England BioLabs), or Tth polymerase (Promega) in previously described reaction mixtures ${ }^{(17)}$ recommended by the manufacturers. For nested PCR, after the initial 5-min denaturation at $95^{\circ} \mathrm{C}$, or, in the case of Vent polymerase, $97^{\circ} \mathrm{C}, 24$ cycles of amplification (the first round of PCR) were conducted. Then, an aliquot of the reaction product was diluted 15 -fold into a fresh reaction mixture containing two new primers (one of which had been radiolabeled with $\left.{ }^{32} \mathrm{P}\right)^{(17)}$ internal to the first pair for 22 more cycles of amplification. Seminested PCR was conducted analogously, but only one of the first two primers was substituted with a nested ${ }^{32} \mathrm{P}$ primer in the second round of PCR. The last primer extension step was for $10 \mathrm{~min}$. The reaction products were analyzed by electrophoresis on a $5 \%$ polyacrylamide gel followed by autoradiography.

\section{RESULTS}

\section{Origin of a Deletion Product during PCR on a Template Containing Direct Alu Repeats 8 kb Apart}

By PCR, we wanted to sensitively detect rare deletion mutations arising in part of 
the human LDLR gene that has many Alu repeat sequences. This gene region displays rearrangements at some of these repeats in patients with familial hypercholesterolemia (FH). ${ }^{(12,18,19)}$ To set up a nested PCR assay to try to detect one mutant in a background of DNA from $10^{6}$ normal cells, as we did previously for a bcl-2 gene translocation, ${ }^{(17)}$ we used primers complementary to sequences 8 $\mathrm{kb}$ apart in the nonmutant LDLR gene to of Alu repeats in intron 15 and exon 18. Because under standard conditions, PCR cannot amplify 8-kb DNA fragments, one might have expected normal human DNA to give no specific product with these primers while DNA containing certain deletions between Alu repeats 3, 4, or 5 in intron 15 and Alu repeats 1,2 , or 3 in exon 18 would (Fig. 1A). As a positive control, DNA from the LDLR partial deletion mutant $\mathrm{FH} 781^{(12)}$ was used as a template. It should give fragments of reveal deletions formed between clusters

0.34 and $0.40 \mathrm{~kb}$ when amplified with primer pairs in $15 \mathrm{c}$ and ex $18 \mathrm{a}$ or in $15 \mathrm{~b}$ and ex18a, respectively, in the second round of PCR (Fig. 1A).

Upon nested or seminested PCR catalyzed by the AmpliTaq Stoffel fragment, similar sized products were obtained from normal human brain DNA (from $\sim 10^{6}$ cells) alone or mixed with a plasmid carrying part of the LDLR gene with the FH781 deletion (100-1000 copies of pLDLR-FH781; Fig. 2, lanes 1, 5, and 9 vs. lanes 4,8 , and 12 ) but not from reactions without template DNA. Similar results were obtained with DNA from human cell lines or recently generated clones (data not shown). Therefore, the deletion was not a contaminant in the DNA preparations but, rather, was generated in vitro by a "jumping reaction"(20) between incompletely extended Alu repeats in which one of the primers is only partially extended through the Alu repeat region and then is used as a longer

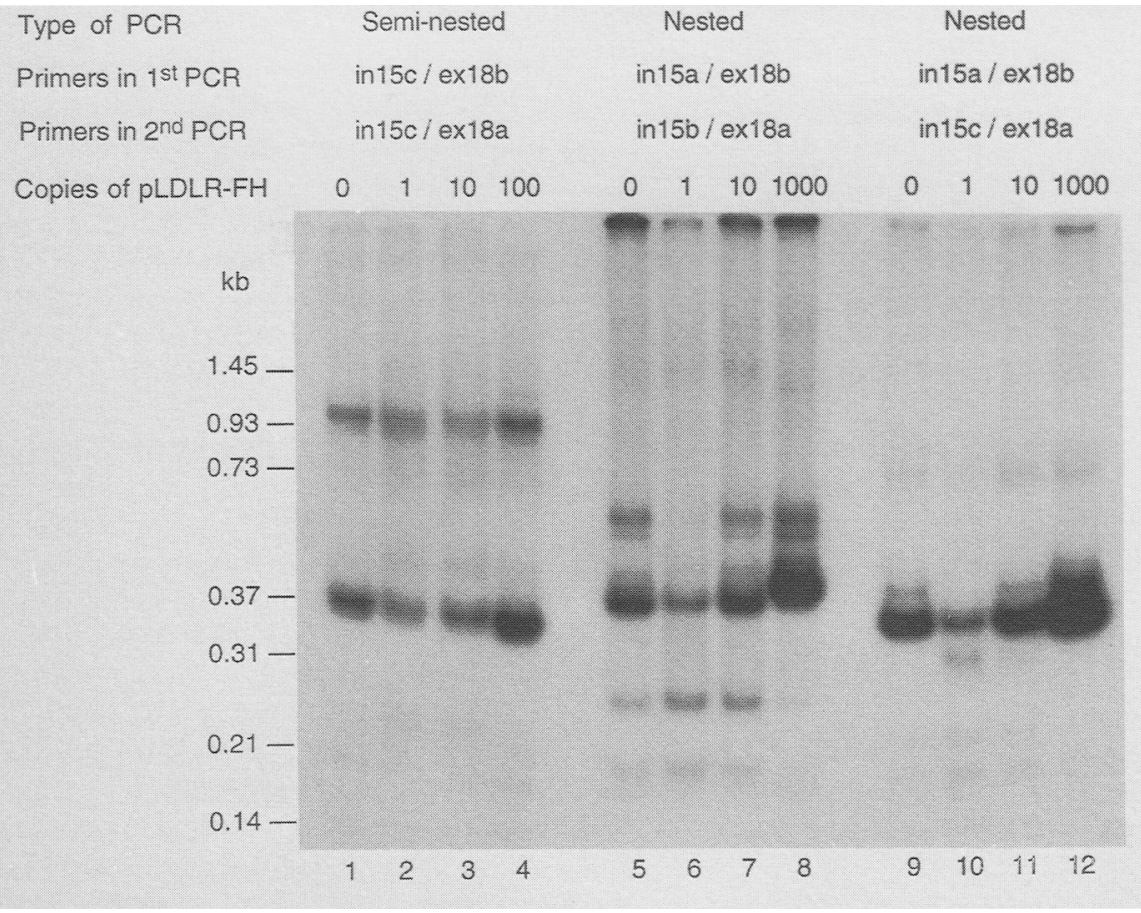

FICURE 2 PCR amplification of an in vivo deletion product and formation of an in vitro deletion (jumping) product from a portion of the LDLR gene containing Alu repeats in the same orientation. The indicated average number of copies of target pLDLR-FH781 molecules (the source of the in vivo deletion product) mixed with $7 \mu \mathrm{g}$ of normal human DNA were amplified with the indicated unlabeled primers for the first round of PCR (each cycle: $94^{\circ} \mathrm{C}$ for $1 \mathrm{~min} ; 60^{\circ} \mathrm{C}$ for $1 \mathrm{~min}$; $72^{\circ} \mathrm{C}$ for $3 \mathrm{~min}$ ) and primers in $15 \mathrm{c}$ and ${ }^{32} \mathrm{P}$-labeled ex18a (lanes $1-4 ; 9-12$ ) or in $15 \mathrm{~b}$ and ${ }^{32} \mathrm{P}$ labeled ex18a (lanes 5-8) in the second round of PCR (same thermocycling conditions but with a $55^{\circ} \mathrm{C}$ annealing temperature) in reactions catalyzed by the AmpliTaq Stoffel fragment. The positions in the gel of molecular-weight markers are indicated. The $\sim 0.34$ - and $0.40-\mathrm{kb}$ bands are the PCR products from the LDLR gene. The other bands and background smears probably come from products involving Alu repeat annealing to the human genomic sequences with the exception of the $\sim 0.9-\mathrm{kb}$ band in lanes $1-4$, that is discussed in the legend to Fig. 3 . primer upon subsequent annealing to the other Alu repeat almost $8 \mathrm{~kb}$ away (Fig. 1B).

When rat DNA was substituted for human DNA, no labeled DNA bands were seen upon PCR in the absence of added pLDLR-FH781 (Fig. 3, lanes 1, 5, 9) because the primers used for PCR of the human gene were not homologous to those of the rat gene. ${ }^{(21)}$ When either AmpliTaq Stoffel fragment or the intact Taq polymerase catalyzed the amplification, the addition of an average of $1-100$ copies of the pLDLR-FH781 plasmid to the rat DNA gave the expected 0.34 - or $0.40-\mathrm{kb}$ bands depending on the primers (Fig. 3; data not shown). The divergence of the rodent $\mathrm{B} 1$ family repeat from the related human Alu repeat ${ }^{(22)}$ probably explains why there were much less smearforming or high-molecular-weight products seen in Figure 3 than in Figure 2. A comparison of the intensity of the specific PCR bands from normal human DNA alone versus from FH781 mutant DNA in the simultaneously conducted amplifications in Figures 2 and 3 indicates that the efficiency of generation of the in vitro deletion product was much lower than the amplification of the FH781 allele given that $\sim 2 \times 10^{6}$ copies of the haploid normal human DNA were present in the former case.

The in vitro deletion product from nested PCR of normal human DNA with the primer pairs in $15 \mathrm{c}$ and ex18a in the second round of amplification was 0.34 $\mathrm{kb}$, the same length as the PCR product from pLDLR-FH781 (Fig. 4A, lanes 4-7 vs. 2). With primers in $15 \mathrm{~b}$ and ex18a in the second round of PCR under stringent annealing conditions, a $0.4-\mathrm{kb}$ band was obtained that coelectrophoresed with the analogous pLDLR-FH781 product (Fig. 4B, lanes 10-12). An additional nonspecific band in this region was seen under less stringent PCR annealing conditions (Fig. 4B, lanes $3,4,7,8$ ). The identity of the 0.34 - and $0.40-\mathrm{kb}$ PCR products obtained from normal human DNA was confirmed by restriction analysis of these products versus those from the FH781 allele (Table 1).

\section{Characterizing Conditions for Formation of the In Vitro Deletion (Jumping) Product from Distant Alu Repeats}

We tried to decrease the jumping reaction by varying PCR conditions. Increas- 


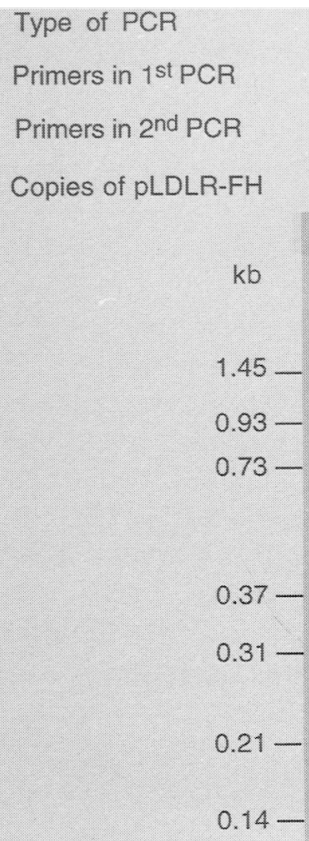

$\mathrm{kb}$

1.45

$0.93-$

$0.73-$

$0.37-$

$0.31-$

$0.21-$

$0.14-$

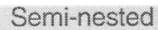

in $15 \mathrm{c} / \mathrm{ex} 18 \mathrm{~b}$

in $15 a /$ ex $18 b$

in $15 b /$ ex18a

$\begin{array}{llll}0 & 1 & 10 & 100\end{array}$

0

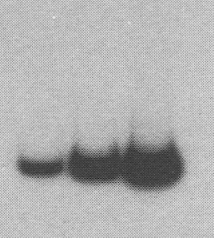

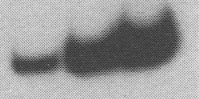

1234
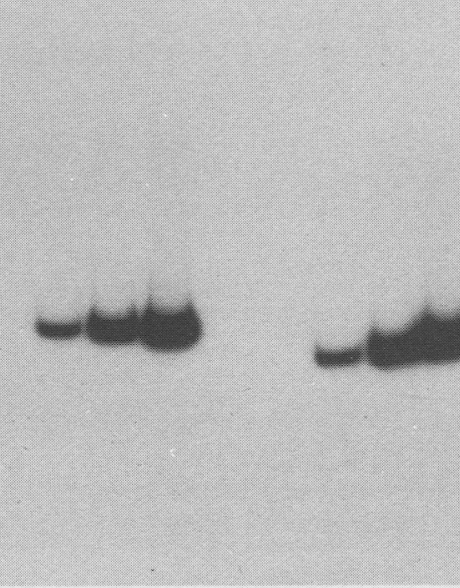

$\sqrt{2}$

$\begin{array}{llll}9 & 10 & 11 & 12\end{array}$

FIGURE 3 PCR amplification of a cloned in vivo LDLR gene deletion product in the presence of a vast excess of rat DNA instead of human DNA. The PCR and analysis were conducted in parallel to that of Fig. 2 and at the same time. The only difference is that $7 \mu \mathrm{g}$ of normal rat DNA was present here instead of $7 \mu \mathrm{g}$ of normal human DNA in addition to an average of 1-100 copies of the deletion mutant pLDLR-FH781 DNA. $\sim 0.9-\mathrm{kb}$ band in lanes $2-4$ might be attributable to mispriming, during two rounds of PCR, by oligonucleotide in $15 \mathrm{c}$ in the FH781 LDLR allele's intron 15 , most of the sequence of which is unknown.

ing the extension time from 3 to $10 \mathrm{~min}$ decreased only slightly the formation of specific PCR products from nonmutant human DNA (Fig. 4B) while it increased the amount of heterogeneously sized DNA (2-20 kb; data not shown) that did not enter a $5 \%$ polyacrylamide gel. The addition of PCR specificity enhàncers (10\% formamide, $5 \%$ or $10 \%$ dimethylsulfoxide, $10^{-5}$ or $10^{-4} \mathrm{M}$ tetramethylammonium chloride) or the predigestion of the template DNA with a restriction endonuclease that cleaved only outside the amplified region also did not appreciably decrease the formation of the in vitro deletion product from nonmutant human DNA (data not shown).

We examined the effect of varying the enzyme used for the amplification on the extent of formation of the in vitrogenerated deletion-type PCR product from normal human DNA. With the AmpliTaq Stoffel fragment or the intact Taq DNA polymerase catalyzing the PCR, the in vitro-generated mutant was seen when $10^{4}$ copies of the diploid genome were in the initial reaction mixture, but no specific product was seen when only $10^{3}$ copies were in the starting reaction
(Fig. 4A, lanes 3,4). This contrasts with the single-molecule sensitivity of detection of the in vivo-generated mutant (Fig. 3). When Vent polymerase or Vent polymerase exo- was used to amplify $10^{5}$ copies of the normal human diploid genome, we observed the formation of the in vitro deletion (jumping) product in some, but not all, replicate reactions. The lack of reproducibility of the jumping reaction with these polymerases probably reflects a lower rate of generation of the primer extension products prematurely terminated within the Alu repeat so that stochastic effects are more apparent than with Taq polymerase or the Stoffel fragment of that enzyme. Although the jumping product was never seen in repeated amplifications of $\sim 10^{6}$ copies $(7 \mu \mathrm{g})$ of the normal human genome with Vent polymerase, this is probably because of the general inhibition of amplification by such high concentrations of nontarget DNA. This type of inhibition was seen, for example, in mixing experiments with pLDLR-FH781 DNA and normal human DNA (data not shown) and may reflect the unusually low $K_{\mathrm{m}}$ of Vent polymerase for DNA. ${ }^{(23)}$
Despite the report that $T$ th polymerase can amplify long stretches of DNA, ${ }^{(24)}$ we saw only the jumping products and background smears of 0.6- to 2-kb DNA and no expected 8-kb band when we amplified $7 \mu \mathrm{g}$ of normal human DNA with this polymerase and the LDLR primers (data not shown).

\section{Lack of Formation of Jumping Products from Alu Repeats or an Oligonucleotide Repeat in Inverted Orientation}

Next, we tested whether a jumping reaction like that described above for the direct Alu repeats of the LDLR gene PCR would occur between inverted Alu repeats (Alu repeats 1 and 2) in intron 2 of the human $\alpha$-gal A gene. ${ }^{(13)}$ The closest borders of these repeats are $145 \mathrm{bp}$ apart (Fig. 5A). As for the PCR of the LDLR gene, unique-sequence primers annealing just beyond these Alu repeats were used to amplify the region $(\sim 0.8-0.9 \mathrm{~kb})$ containing these repeats in nested or seminested PCR. In the Taq polymerasecatalyzed reaction on normal human DNA, the $\alpha$-gal gene was amplified so that we could detect as few as an average of two molecules of the $\alpha$-gal-containing DNA per sample (data not shown).

No jumping products were seen in the case of the Alu repeats in the $\alpha$-gal gene even when $2 \times 10^{4}$ or $2 \times 10^{6}$ copies of the gene were present (Fig. 5B; data not shown). If such a product had formed during seminested PCR of the $\alpha$-gal gene using ${ }^{32} \mathrm{P}$-labeled primer $\mathrm{b}$ and unlabeled primer $d$ in the second round of the amplification, then it should have been visible in autoradiographs as a $0.81-\mathrm{kb}$ jumping product in addition to the expected $0.87-\mathrm{kb}$ standard PCR product. A 0.81-kb jumping product could have arisen, for example, by incomplete extension of primer $b$ annealed $52 \mathrm{bp}$ upstream of Alu repeat 1 in intron 2 followed by its annealing via the Alu repeat to the complementary strand of Alu repeat 2, which is in the opposite orientation to Alu repeat 1 (Fig. 5A). As shown in Figure 5B (lanes 2,4), no minor 0.81$\mathrm{kb}$ band (jumping product) was seen in these samples amplified with primers $b$ and $d$, whereas the expected major 0.81 $\mathrm{kb}$ product (normal PCR product) was obtained when the same DNA was amplified with primers $b$ and $c$.

Furthermore, if radiolabeled jumping products formed upon PCR with radiola- 


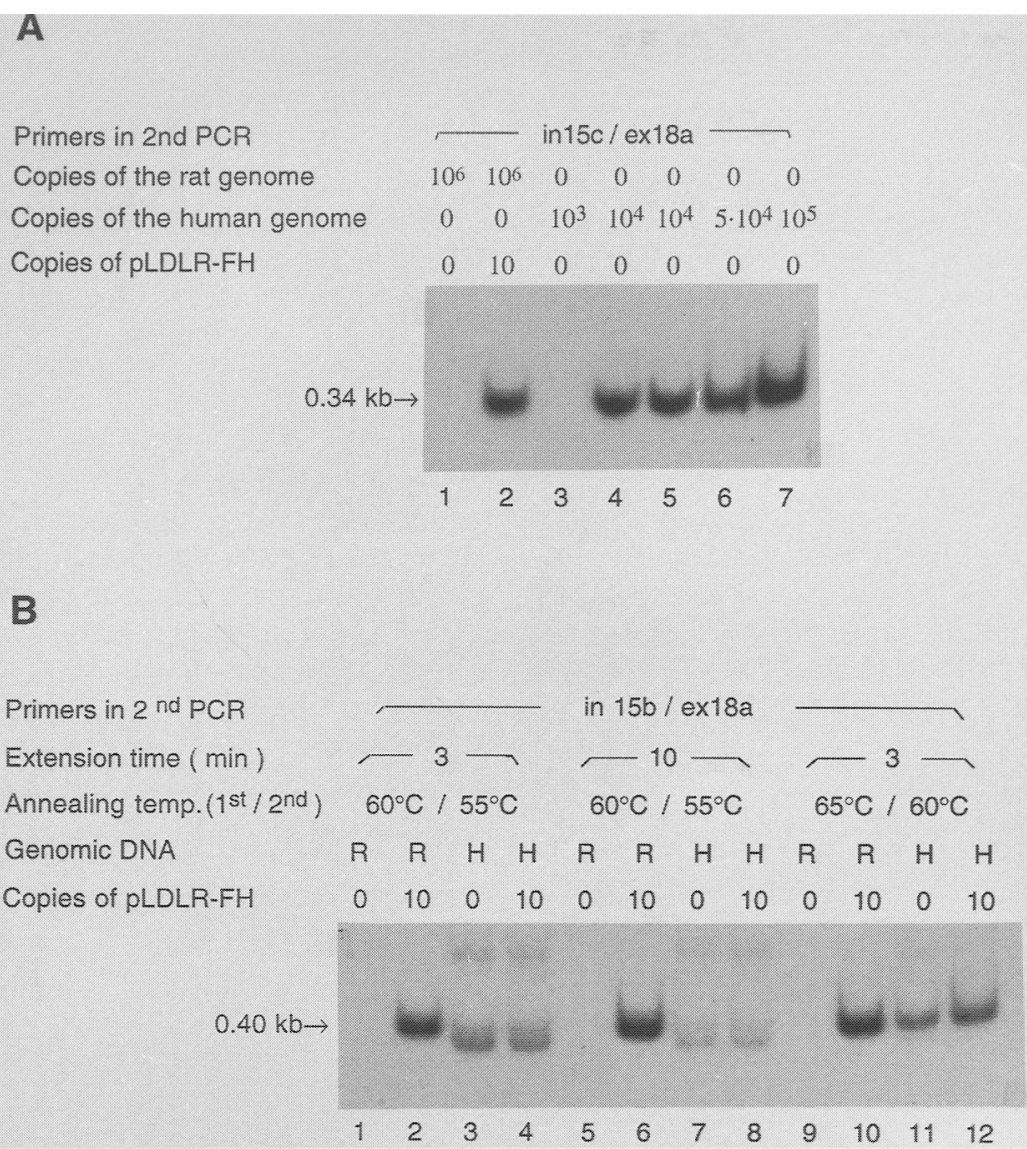

FIGURE 4 Effect of varying the concentration of the normal human DNA or the annealing temperature for PCR on the formation of the in vitro deletion (jumping) product. These reactions were catalyzed by the Stoffel fragment of AmpliTaq, but similar results were obtained with the intact Taq polymerase. The primers used in the first round of PCR were in $15 \mathrm{a}$ and ex18b. $(A)$ The in vitro deletion product from PCR of the normal human diploid genome (lanes 3-7; the number of copies of the diploid genome are indicated) and the PCR product from the analogous in vivo deletion mutant (pLDLR-FH781; lane 2 ). (Lanes 1,2$)$ Only rat DNA $\left(10^{6}\right.$ copies of the diploid genome, $7 \mu \mathrm{g}$ ) or rat DNA plus pLDLR-FH781 was present. $(B)$ The annealing conditions and the time for primer extension were varied for the first and second round of PCR. The conditions used in $A$ were those specified in lanes $1-4$ of $B$. The indicated number of copies of pLDLR-FH781 was mixed with rat DNA or human DNA $\left(10^{5}\right.$ copies of the normal diploid genome), $\mathrm{R}$ and $\mathrm{H}$, respectively. The sizes of the specific PCR products are shown. Only the portion of the gels containing these products is included here. The band below the $0.4-\mathrm{kb}$ band in $B$ (lanes $3,4,7,8$ ) is attributable to a nonspecific PCR product.

beled primer $b$ and either unlabeled primer $\mathrm{d}$ or $\mathrm{c}$, these jumping products should be distinguishable from the expected PCR products by restriction analysis. Sau96I has a cleavage site midway within Alu repeat 2 of intron 2 of the $\alpha$-gal gene, but no Sau96I site is present in Alu repeat 1 (Fig. 5A). Without the jumping reaction, the only radiolabeled product from Sau96I digestion following PCR with either set of primers should be $597 \mathrm{bp}$ long. If primer $b$, which is adjacent to Alu repeat 1 , is incompletely extended through that Alu repeat and then is used as a primer by annealing to Alu repeat 2, the Sau96I digestion should give either an additional radiolabeled 216- or 813-bp band depending on whether primer extension had terminated in the first or the second half of Alu repeat 1 (Fig. 5A). No band was seen in either position of the electrophoresis gel in the digested PCR product samples (Fig. 5B, lanes 5-8), and the level of radioactivity in these regions indicated that if such jumping products formed they constituted $<5 \%$ of the expected product.

When inverted repeats are present not too far apart from one another, the denatured strands might form a foldback structure allowing replication through it to give a deletion of the intervening sequences and one copy of the repeat. No evidence for the formation of products with the predicted size for loopout molecules was seen upon amplifying the $\alpha$-gal gene as described above. We also tested as a template a recombinant plasmid DNA (pMKIVdel) that contained as an inverted repeat a 30-bp sequence from the HSV TK gene with the two copies of the sequence only $28 \mathrm{bp}$ apart. Upon $\mathrm{Taq}$ polymerase-catalyzed amplification of $10^{4}-10^{6}$ copies of this plasmid for 35 cycles, only the expected sized product $(0.38 \mathrm{~kb})$ was seen (data not shown). There was no evidence for the formation of a loop-out product $(0.30$ $\mathrm{kb})$ nor for jumping products $(0.17$ or $0.59 \mathrm{~kb}$ ).

\section{DISCUSSION}

In this study we amplified regions of the human genome containing Alu repeats using unique sequence primers rather than consensus sequences for the Alu repeat. ${ }^{(25)}$ During PCR on a part of the nonmutant human LDLR gene containing a pair of direct Alu repeats $\sim 8 \mathrm{~kb}$ apart, a jumping reaction product (Fig. $1 B$ ) was the main amplified DNA fragment when at least $10^{4}$ copies of the gene were present as the template. In addition to the formation of discrete jumping products from the LDLR gene, it appears that the extremely high number of copies of the Alu repeat spread throughout the human genome may be contributing to the formation of a heterodisperse collection of jumping products from Alu repeats not in this region of the LDLR gene (Fig. 2 vs. Fig. 3). Under standard PCR conditions the full-length, 8-kb product would not be expected to form while the jumping product $(0.3-$ $0.4 \mathrm{~kb}$, depending on the primers used) is small enough to be amplified very efficiently. The jumping reaction involves truncation of primer extension, which can occur to a considerable extent as a side reaction during PCR. ${ }^{(26)}$ The jumping product from the LDLR gene was indistinguishable from an in vivo deletion product with its borders within direct Alu repeats in intron 15 and in exon 18 (Fig. 1A). In contrast, no jumping product was detected from two inverted Alu repeats that are $145 \mathrm{bp}$ apart in intron 2 of the human $\alpha$-gal A gene (Fig. 5).

These results can be explained by the jumping product forming in large 
TABLE 1 Restriction Analysis of In Vitro Deletion (Jumping) PCR Products

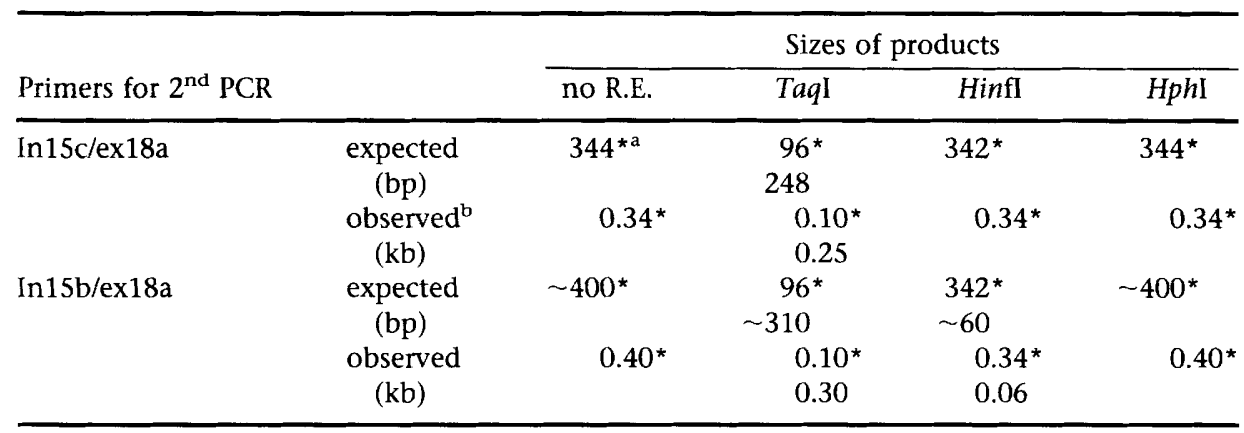

Cleavage of the products from nested PCR of normal human DNA with the indicated LDLR gene primers (Fig. 1) in the second round of PCR and primers in $15 \mathrm{a}$ and ex $18 \mathrm{~b}$ in the first round. There is one TaqI cleavage site but no HphI site in the Alu repeat between these primers in FH781 or in the equivalent in vitro jumping product (Fig. 1). The one Hinfl site in this amplified region is within the in $15 \mathrm{c}$ primer.

${ }^{\text {aT }}$ The fragment sizes marked with an asterisk $\left(^{\star}\right)$ are those expected or found to be radiolabeled when ${ }^{32}$ P-labeled primer ex18a was used in the second round of PCR. The expected sizes of the PCR, or PCR + restriction products were calculated from the sequence of the FH781 deletion mutant.

${ }^{b}$ PCR products were visualized by ethidium bromide-induced fluorescence (unlabeled products) or autoradiography (labeled products). amounts when there is no efficient competing reaction. This competing reaction, in the case of $\alpha$-gal gene PCR with its close Alu repeats, could involve annealing of oligonucleotide primers present in great excess and their rapid extension even at the $55^{\circ} \mathrm{C}$ or $50^{\circ} \mathrm{C}$ temperature used for the 1-min "annealing" stage in the amplification as is seen in two-temperature PCR. This would decrease the concentration of template available for annealing via the Alu repeat sequences to the low concentration of incompletely extended primers ending within the Alu repeat region. In contrast, in the LDLR gene PCR with its distant Alu repeats, these Alu repeats would be available for annealing to incompletely extended primers in the jumping reaction because the large distance between the oligonucleotide primer binding sites
A

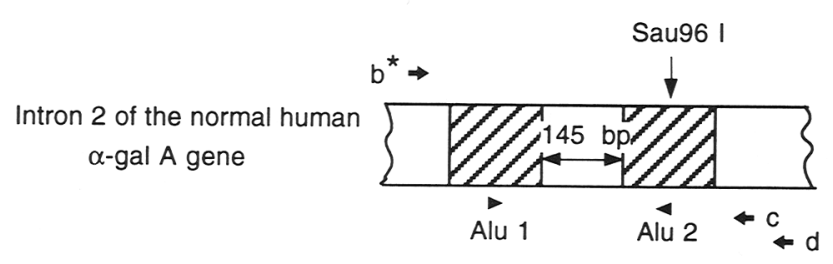

Radiolabeled PCR products from the second round of PCR

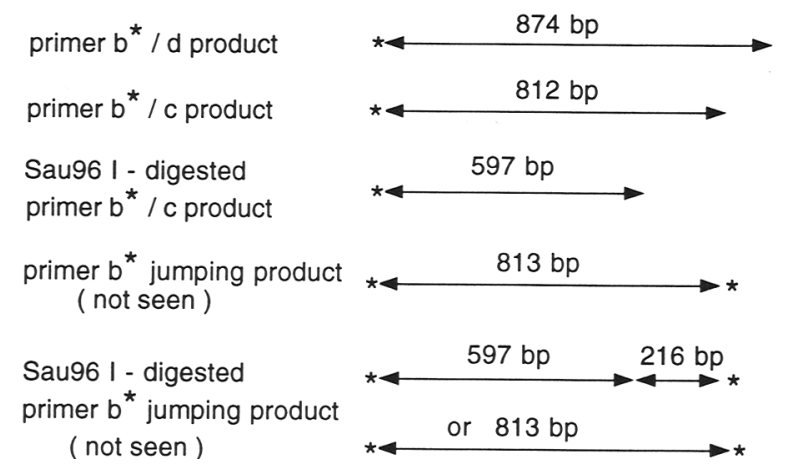

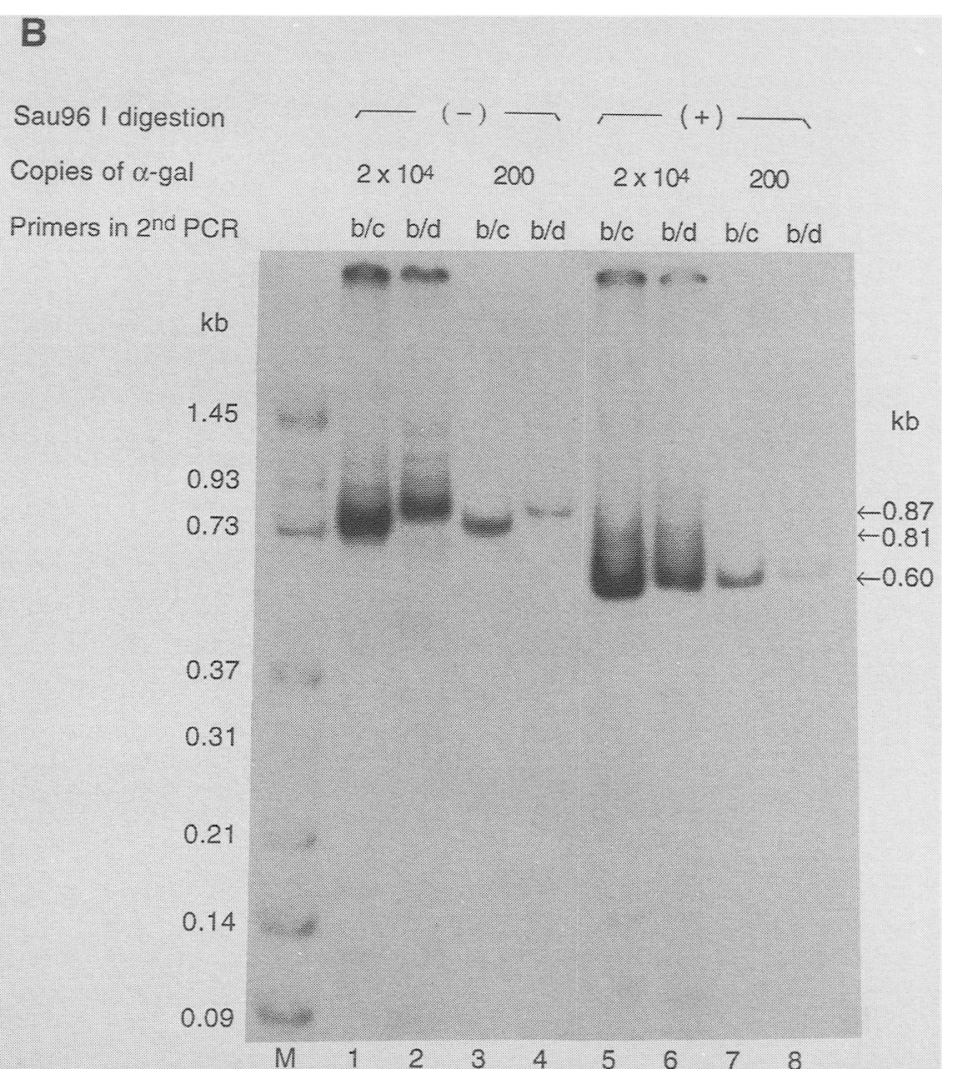

FIGURE 5 PCR products from intron 2 of the $\alpha$-gal A gene containing Alu repeats in inverted orientation and the restriction analysis of these products. $(A)$ The amplified portion of the $\alpha$-gal A gene and its expected or observed PCR products (not to scale). The conditions for PCR were the same as those of Fig. 2 except that the intact Taq polymerase catalyzed the reaction, and the annealing temperatures for the first and second rounds were $55^{\circ} \mathrm{C}$ and $50^{\circ} \mathrm{C}$, respectively. The locations of the two Alu repeat sequences in this region are diagrammatically depicted by hatched bars, their orientation by solid arrowheads, and the Sau $96 \mathrm{I}$ site present in only one of these repeats is shown by a vertical arrow. The PCR primers are indicated by short arrows, and the radiolabeled primer is marked with an asterisk( $\left.{ }^{*}\right)$. The sizes of the PCR products and digested PCR products are given. $(B)$ The lack of formation of jumping products from the above-mentioned Alu repeats in the human $\alpha$-gal gene. For the first round of PCR, unlabeled primers a and $\mathrm{d}$ were present, and in the second round the indicated primer pairs were used with primer b as the radiolabeled primer in Taq polymerase-catalyzed reactions. The indicated number of copies of the human $\alpha$-gal gene were provided by the corresponding number of copies of the haploid human genome. (Lanes 5-8) The Sau96I digestion of PCR products of the samples from lanes 1-4. The sizes of the specific radiolabeled PCR products or Sau96I-digested PCR product and of molecular-weight markers are given. 
flanking the direct Alu repeats would inhibit the template from becoming double-stranded by normal oligonucleotide primer extension in the region where the two partially homologous Alu repeats are to anneal in a jumping reaction (Fig. 1B).

Alu repeats typically are head-to-tail dimers, with the directly repeated monomers referred to as left and right monomers (Fig. 1). ${ }^{(6)}$ The jumping reaction that we saw during PCR amplification of the human LDLR gene may have taken place from the left monomer of Alu repeat 3 in intron 15 to the left monomer of Alu repeat 3 in exon 18 or between the corresponding right monomers. A jumping reaction might also have occurred from the right half of either of these Alu repeats or of the hybrid Alu repeat in pLDLR-FH781 to the left half of the same repeat or vice versa. However, such a reaction was not evident as seen by the absence of jumping products of the size expected from such a left-half Alu repeat to right-half Alu repeat jump when amplifying pLDLR-FH781 (Fig. 3) despite the presence of a 51-bp region with $76 \%$ homology between the two halves of the amplified Alu repeat in pLDLR-FH781. Therefore, we conclude that even when a region containing two adjacent direct repeats is subject to PCR, if the oligonucleotide primer-binding sites are close enough together (in this case, $0.3-0.4 \mathrm{~kb}$ apart), the jumping reaction may not be seen. This may be attributable to effective competition of the jumping reaction by the amplification reaction producing the full-length product as described above.

On the other hand, we did see a ladder of jumping products when amplifying part of the oriP region of the Epstein Barr virus genome (data not shown), which contains 20 tandem repeats of a 30 -bp sequence. ${ }^{(27)}$ This PCR was conducted with unique sequence primers outside this repeat region under similar conditions used for amplification of the LDLR and $\alpha$-gal sequences. Such ladders due to PCR side reactions have been seen with minisatellite DNAs and with oligonucleotide repeats implicated in the control of gene expression and genetic imprinting. ${ }^{(28)}$ The ladder of bands from the oriP sequence appears to be derived from multiple jumping reactions between the 30-bp repeats. The ratio of DNA in this ladder to DNA in the fulllength PCR product of $0.7 \mathrm{~kb}$ was $\sim 0.4$.
Therefore, in this case, large amounts of jumping products formed during PCR despite the competing amplification of the full-length reaction product. This large amount of jumping product formation is probably the result of the presence of numerous tandem repeats, which increases the likelihood of annealing at the 3 ' end of an incompletely extended primer.

\section{ACKNOWLEDGMENTS}

We thank Michael Greene for his participation in preliminary experiments. Fellowship support for W.J. and G.Q. was provided in part by the Tulane Cancer Center and the National Institute of Environmental Health Sciences/Superfund Interdisciplinary Doctoral Funds, respectively. This research was supported in part by the National Institutes of Health (ESO5946) and the Department of Defense through the Center for Bioenvironmental Studies at Tulane University.

\section{REFERENCES}

1. Saiki, R.K., D.H. Gelfand, S. Stoffel, S.J. Scharf, R. Higuchi, G.T. Horn, K.B. Mullis, and H.A. Erlich. 1988. Primer-directed enzymatic amplification of DNA with a thermostable DNA polymerase. Science 239: $487-491$.

2. Shuldiner, A.R., A. Nirula, and J. Roth. 1989. Hybrid DNA artifact from PCR of closely related target sequences. Nucleic Acids Res. 17: 4409.

3. Meyerhans, A., J.-P. Vartanian, and S. Wain-Hobson. 1990. DNA recombination during PCR. Nucleic Acids Res. 18: 16871691.

4. Jeffreys, A.J., V. Wilson, R. Neumann, and J. Keyte. 1988. Amplification of human minisatellites by the polymerase chain reaction: Towards DNA fingerprinting of single cells. Nucleic Acids Res. 16: 1095310971.

5. Cariello, N.F., W.G. Thilly, J.A. Swenberg, and T.R. Skopek. 1991. Deletion mutagenesis during polymerase chain reaction: Dependence on DNA polymerase. Gene 99: 105-108.

6. Schmid, C.W. and W.R. Jelinek. 1982. The Alu family of dispersed sequences. Science 216: 1065-1070.

7. Kariya, Y., K. Kikuya, Y. Hayashizaki, S. Himeno, S. Tarui, and K. Matsubara. 1987. Revision of consensus sequence of human Alu repeats: A review. Gene 53: 110.

8. Nicholls, R.D., N. Fischel-Ghodsian, and D.R. Higgs. 1987. Recombination at the human $\alpha$-globin gene cluster: Sequence features and topological constraints. Cell 49: 369-378.

9. Markert, M.L., J.J. Hutton, D.A. Wiginton, J.C. States, and R.E. Kaufman. 1988. Adenosine deaminase (ADA) deficiency due to deletion of the ADA gene promoter and first exon by homologous recombination between two Alu elements. J. Clin. Invest. 81: 1323-1327.

10. Ariga, T., P.E. Carter, and A.E. Davis, III. 1990. Recombinations between Alu repeat sequences that result in partial deletions within the $\mathrm{C} 1$ inhibitor gene. $\mathrm{Ge}$ nomics 8: $606-613$.

11. Papadopoulos, P.C., A.M. Greenstein, R.A. Gaffney, C.A. Westbrook, and L.M. Wiedemann. 1990. Characterization of the translocation breakpoint sequences in Philadelphia-positive acute lymphoblastic leukemia. Genes, Chrom. Cancer 1: 233-239.

12. Lehrman, M.A., D.W. Russell, J.L. Goldstein, and M.S. Brown. 1987. Alu-Alu recombination deletes splice acceptor sites and produces secreted low density lipoprotein receptor in a subject with familial hypercholesterolemia. J. Biol. Chem. 262: 3354-3361.

13. Kornreich, R., R.J. Desnick, and D.F. Bishop. 1989. Nucleotide sequence of the human $\alpha$-galactosidase A gene. Nucleic Acids Res. 17: 3301.

14. Ehrlich, M., M.A. Gama-Sosa, L.-H. Huang, R.M. Midgett, K.C. Kuo, R.A. McCune, and C. Gehrke. 1982. Amount and distribution of 5-methylcytosine in human DNA from different types of tissues or cells. Nucleic Acids Res. 10: 2709-2721.

15. Stuart, G.W., P.F. Searle, H.Y. Chen, R.L. Brinster, and R.D. Palmiter. 1984. A 12 base-pair DNA motif that is repeated several times in metallothionein gene promoters confers metal regulation a heterologous gene. Proc. Natl. Acad. Sci. 81: 7318-7322.

16. Kornreich, R., D.F. Bishop, and R.J. Desnick. 1990. $\alpha$-Galactosidase A gene rearrangements causing Fabry disease. J. Biol. Chem. 265: 9319-9326.

17. Zhang, X.-Y. and M. Ehrlich. 1994. Detection and quantitation of low numbers of chromosomes containing bcl-2 oncogene translocations using semi-nested PCR. BioTechniques 16: 502-507.

18. Horsthemke, B., U. Beisiegel, A. Dunning, J.R. Havinga, R. Williamson, and S. Humphries. 1986. Unequal crossing-over between two Alu-repetitive DNA sequences in the low-density-lipoproteinreceptor gene. Eur. J. Bioch. 164: 77-81.

19. Lehrman, M.A., J.L. Goldstein, D.W. Russell, and M.S. Brown. 1987. Duplication of seven exons in the LDL receptor gene caused by Alu-Alu recombination in a subject with familial hypercholesterolemia. Cell 48: 827-835.

20. Huang, L.-M. and K.-T. Jeang. 1994. Long- 
range jumping of incompletely extended polymerase chain fragments generates unexpected products. BioTechniques 16: 242-246.

21. Hoffer, M.J.V., M.M. van Eck, F. Petrij, A. van der Zee, E. de Wit, D. Meijer, G. Grosveld, L.M. Havekes, M.H. Hofker, and R.R. Frants. 1993. The mouse low density lipoprotein receptor gene: cDNA sequence and exon-intron structure. Biochem. Biophys. Res. Commun. 191: 880-886.

22. Haynes, S.R., T.P. Toomey, L. Leinwand, and W.R. Jelinek. 1981. The Chinese hamster Alu-equivalent sequence: A conserved, highly repetitious, interspersed deoxyribonucleic acid sequence in mammals has a structure suggestive of a transposable element. Mol. Cell. Biol. 1: 573583.

23. Kong, H., R.B. Kucera, and W.E. Jack. 1993. Characterization of a DNA polymerase from the hyperthermophile $A r$ chaea Thermococcus litoralis. J. Biol. Chem. 268: 1965-1975.

24. Cohen, J. 1994. "Long PCR" leaps into larger DNA sequences. Science 263: 1564 1565.

25. Nelson, D.L., S.A. Ledbetter, L. Corbo, M.F. Victoria, R. Ramirez-Solis, T.D. Webster, D.H. Ledbetter, and C.T. Caskey. 1989. Alu polymerase chain reaction: A method for rapid isolation of human-specific sequences from complex DNA sources. Proc. Natl. Acad. Sci. 86: 66866690.

26. Olsen, D.B. and F. Eckstein. 1989. Incomplete primer extension during in vitro DNA amplification catalyzed by Taq polymerase; exploitation for DNA sequencing. Nucleic Acids Res. 17: 9613-9620.

27. Lupton, S. and A. Levine. 1985. Mapping genetic elements of Epstein-Barr virus that facilitate extrachromosomal persistence of Epstein-Barr virus-derived plasmids in human cells. Mol. Cell. Biol. 5: 2533-2542.

28. Knight, S.J.L., A.V. Flannery, M.C. Hirst, L. Campbell, Z. Christodoulou, S.R. Phelps, J. Pointon, H.R. Middleton-Price, A. Barnicoat, M.E. Pembrey, J. Holland, B.A. Oostra, M. Bobrow, and K.E. Davies. 1993. Trinucleotide repeat amplification and hypermethylation of a $\mathrm{CPG}$ island in FRAXE mental retardation. Cell 74: 127134.

Received April 23, 1994; accepted July 8, 1994. 


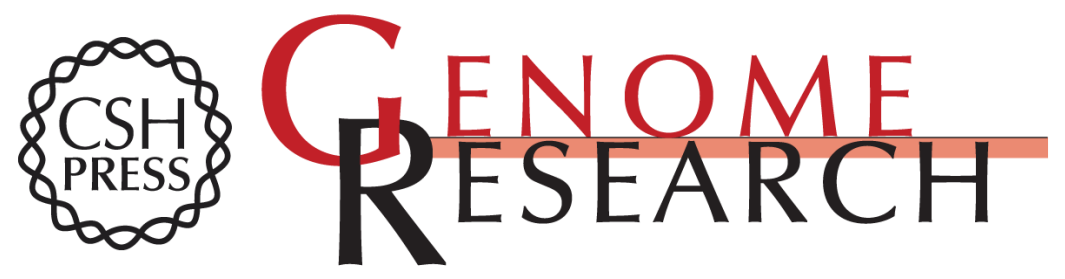

\section{Effect of internal direct and inverted Alu repeat sequences on PCR.}

W Ji, X Y Zhang, G S Warshamana, et al.

Genome Res. 1994 4: 109-116

References This article cites 28 articles, 10 of which can be accessed free at:

http://genome.cshlp.org/content/4/2/109.full.html\#ref-list-1

\section{License}

Email Alerting Receive free email alerts when new articles cite this article - sign up in the box at the Service top right corner of the article or click here.

\section{Affordable, Accurate Sequencing.}

To subscribe to Genome Research go to: https://genome.cshlp.org/subscriptions 